\title{
GMR
}

\section{Role of Th1/Th2 cytokines in the diagnosis and prognostic evaluation of ankylosing spondylitis}

\author{
J.T. Wen', D.H. Zhang', P.F. Fang' ${ }^{2}$, M.H. Li ${ }^{2}$, R.J. Wang ${ }^{2}$ and S.H. Li ${ }^{1}$ \\ ${ }^{1}$ Department of Orthopedics, TCM Hospital of Gansu Province, Lanzhou, \\ Gansu, China \\ ${ }^{2}$ Department of Orthopedics, the Baiyin branch of TCM Hospital of Gansu Province, \\ Lanzhou, Gansu, China \\ Corresponding author: D.H. Zhang \\ E-mail: dehongzhangf@sina.com \\ Genet. Mol. Res. 16 (1): gmr16019322 \\ Received September 20, 2016 \\ Accepted December 12, 2016 \\ Published March 16, 2017 \\ DOI http://dx.doi.org/10.4238/gmr16019322
}

Copyright $(92017$ The Authors. This is an open-access article distributed under the terms of the Creative Commons Attribution ShareAlike (CC BY-SA) 4.0 License.

\begin{abstract}
Ankylosing spondylitis (AS), a progressive disease of the spine, manifests as peripheral arthritis with tendon and ligament inflammation that restricts activity. AS is a rheumatoid autoimmune disease although the rheumatoid factor is absent in patients with AS. It is characterized by inflammatory changes such as elevated levels of serum inflammatory factors. The roles of Th1 and Th2 cytokines in autoimmune diseases are well known. However, the roles of these cytokines in the diagnosis and prognosis of AS is poorly understood. The aim of this study was to investigate the roles of Th1/Th2 cytokines in the diagnosis and prognosis of AS. The BASDAI activity, BASFI functional index, BASMI measurement score, and the levels of CRP and ESR were measured during the treatment of patients with active AS. The levels of IFN- $\gamma$ and TNF- $\alpha$ (Th1 cytokines) and IL-4 and IL-10 (Th2 cytokines) were quantified. The levels of IL-4 and IL-10 were significantly low in the serum of patients with active AS, who
\end{abstract}

Genetics and Molecular Research 16 (1): gmr16019322 
also had high IFN- $\gamma$ and TNF- $\alpha$ levels compared to those in the control individuals $(\mathrm{P}<0.05)$. After treatment, the levels of IL-4 and IL-10 increased while those of IFN- $\gamma$ and TNF- $\alpha$ decreased compared to those in individuals with active AS $(\mathrm{P}<0.05)$. The disease activity index correlated positively with levels of IFN- $\gamma$ and TNF- $\alpha$ and negatively with levels of IL-4 and IL-10, but not with that of CRP or ESR. Changes in the levels of Th1/2 cytokines in patients with AS may reflect disease activity and prognosis.

Key words: Ankylosing spondylitis; Th1; Th2; Inflammatory cytokines

\section{INTRODUCTION}

Ankylosing spondylitis (AS) is an autoimmune disease that is considered to be a rheumatoid disorder albeit with the absence of rheumatoid factors (Almasi et al., 2016). AS is a familial inherited disease involving multiple gene loci, among which, the HLA-B27 locus has been shown to have a strong association with the disease. Patients with AS have strong sex preference, as it occurs frequently in young males in the age group of 20-30 years (Lindström et al., 2016b; Zou et al., 2016). Although AS has an insidious onset and slow progression, no effective treatment has been found, which severely affects the efficacy of treatment (Fischer et al., 2015; Kisacik et al., 2016a). AS can affect the tissues of the spine and major joints of the body, which elicit an inflammatory response (Colombo et al., 2015; Hoxha et al., 2016). In addition, AS can also affect major organs such as the lungs besides causing stiffness of joints (Arikan et al., 2016; Qian et al., 2016). Currently, knowledge about AS pathogenesis is limiting; however, genetic, environmental, and immune factors are speculated to be involved.

The T helper cells (Th cells), a type of T cell, play an important role in the regulation of immune response. These Th cells can be further divided into Th1 and Th2 cells, and the imbalance of cytokines secreted by the Th1 and Th2 cells is critical for the pathogenesis and progression of autoimmune diseases (Roberts et al., 2016). Th1 cells secrete cytokines such as IFN- $\gamma$ and TNF- $\alpha$, which facilitate macrophage-induced anti-infection and may lead to autoimmune diseases in certain organs (Gracey et al., 2016; Wang et al., 2016). In contrast, Th2 cells secrete cytokines such as IL-4 and IL-10, which promote proliferation, differentiation, antibody production, and participation in humoral immune response of $\mathrm{B}$ lymphocytes. The abnormal expression of Th2 cytokines is correlated with diseases such as asthma (Bautista-Caro et al., 2014; Wang et al., 2015). Therefore, the balance of Th1/Th2 cells determines the response of the immune system. However, the role of Th1/Th2 cytokines in the diagnosis and prognostic evaluation of patients with AS remains poorly understood. The aim of this study was to investigate the role of Th1/Th2 cytokines in diagnosis and prognosis of AS.

\section{MATERIAL AND METHODS}

\section{General information}

Sixty patients, 43 males and 27 females in the age group of 18-36 years (average

Genetics and Molecular Research 16 (1): gmr16019322 
age $=21.0 \pm 4.1$ years) and in the active stage of AS, were recruited to the TCM Hospital of Gansu Province, from December 2014 to June 2015. The patients' conditions were evaluated according to the standard procedure for diagnosis stipulated by the American College of Rheumatology by at least two rheumatologists.

\section{Inclusion criteria}

Patients, whose conditions were in accordance with one or more clinical/imaging standards, were included in the study. The clinical standards included limited lumbar vertebral activity, wrist pain or morning stiffness for at least three months without relief after rest, and lower chest activity. Imaging diagnostic criteria included bilateral grade II (or above) or unilateral grade III or IV sacroiliac articulations with arthritic changes.

\section{Exclusion criteria}

Patients who had a recent episode of inflammation accompanied by the presence of a tumor or injury to the liver or kidney, chronic inflammation, severe cardio-cerebrovascular disease, blood or endocrine disorders, and other rheumatoid diseases or were pregnant or breastfeeding, were excluded from the study. The bath ankylosing spondylitis disease activity index (BASDAI) score was used to identify patients in the active stage of AS (Yu et al., 2015), with patients scoring above 3 being classified as carriers of the active disease.

A cohort of 30 healthy volunteers (22 males and 7 females in the age group of 21-30 years, average age $=20.0 \pm 5.4$ years) was selected as controls. The exclusion criteria were the presence of autoimmune diseases or a family history of chronic disease. This study has been pre-approved by the ethical committee of the TCM Hospital, Gansu Province. Informed consent was obtained from all the participants before commencement of the study.

\section{Patient treatment}

Patients with active AS received non-steroidal anti-inflammatory medicine, including loxoprofen sodium (60 mg, 3 times daily), sulfasalazine (500 mg, 3 times daily for antirheumatoid treatment), and methylprednisolone $(2-4 \mathrm{mg} / \mathrm{kg} / \mathrm{day}, i v$ drip, 3 days per course, 3 courses in total with 4 days interval). After 21 days of treatment, erythrocyte sedimentation index (ESR), $\mathrm{C}$ reactive protein (CRP), and liver/kidney functions were tested, along with estimation of the BASDAI score for disease activity, the BASFI (Bath Ankylosing Spondylitis Functional Index) functional index, and the BASMI (Bath Ankylosing Spondylitis Metrology Index) measurement index.

\section{Disease activity index analysis}

The BASDAI score was used to analyze the disease activity index of AS (Yu et al., 2015) The VAS (Visual Analogue Scale) approach was used to evaluate the extent of pain in the diseased joints of patients with AS, such as pain in the neck, back, and hip, swelling of other joints, and conditions of fatigue and sleep. The morning stiffness score was evaluated based on the time and severity of conditions in a score range from 1 to 10, where a higher score represented higher activity of patients.

Genetics and Molecular Research 16 (1): gmr16019322 


\section{BASMI measurement index}

The measurement index of AS was according to BASMI score (Liang et al., 2015). It recorded four vertebral activity tasks such as pillow wall distance, Schober test, finger pitch, and neck rotation. In each item, 2 points represent severely restricted behavior while 0 point indicates normal activity. The BASMI score was the summation of the scores from all 4 behavioral tasks. Thus, higher BASMI score suggested more severe restriction of vertebral activity.

\section{BASFI functional index}

The BASFI was used to evaluate 10 AS-specific behavioral tasks such as picking up of a pen, wearing a sock (Lindström et al., 2016a). Each task was scored on a scale of 0-10, in which 10 represented severe restriction and 0 represented normal behavior. The BASFI function index was the summation of the scores of all 10 activities. Higher scores indicated severely affected daily lives.

\section{Clinical information of patients}

Clinical symptoms and bodily signs of the patients were documented along with their family history and results from laboratory tests for CRP and ESR.

\section{Sample collection}

Heparin sodium anti-coagulation tubes were used to collect $5 \mathrm{~mL}$ venous blood from patients and healthy controls who had fasted. After centrifugation at $2000 \mathrm{rpm}$ for $10 \mathrm{~min}$, the supernatant was saved at $-20^{\circ} \mathrm{C}$ for further use.

\section{Estimation of cytokine expression by ELISA}

ELISA was used to quantify the expression of Th1 cytokines, IFN- $\gamma$ and TNF- $\alpha$, and Th2 cytokines, IL-4 and IL-10, in the 12 serum samples. In brief, peripheral blood samples were centrifuged to obtain the serum. Optical density (OD) values were measured in a microplate reader according to the manufacturer's instructions of the ELISA kit (Human Th1/Th2/Th17 Panel) (R\&D, Minneapolis, MN, USA). OD values of standards were used to plot the reference curve, from which the levels of Th1 and Th2 cytokines were calculated.

\section{Statistical analysis}

All data were processed with the SPSS 22.0 software. Data are reported as mean \pm standard deviation (SD). The comparison among multiple groups was carried out using the one-way analysis of variance (ANOVA). The student $t$-test was used for paired comparison. Pearson analysis was used to detect the correlation between parameters. A significant level was defined as $\alpha=0.05$. A statistical significance was identified as $\mathrm{P}<0.05$.

Genetics and Molecular Research 16 (1): gmr16019322 


\section{RESULTS}

\section{ESR and CRP levels before and after treatment of AS}

We analyzed the clinical and lab indexes, especially ESR and CRP levels, of patients with active AS, patients receiving treatment for AS, and healthy individuals. Results showed that treatment decreased levels of ESR $(31.63 \pm 2.37 \mathrm{~mm} / \mathrm{h})$ and CRP $(22.34 \pm 3.09 \mathrm{mg} / \mathrm{L})$ when compared to the levels before the treatment $(32.53 \pm 3.36 \mathrm{~mm} / \mathrm{h}$ for ESR and $23.26 \pm$ $3.35 \mathrm{mg} / \mathrm{L}$ for CRP), albeit without significant difference (Table 1).

\section{Table 1. ESR and CRP levels of patients with active AS before and after treatment.}

\begin{tabular}{l|c|c|c}
\hline Index & Control & Before treatment & After treatment \\
\hline ESR $(\mathrm{mm} / \mathrm{h})$ & NA & $32.53 \pm 3.36$ & $31.63 \pm 2.37$ \\
\hline $\mathrm{CRP}(\mathrm{mg} / \mathrm{L})$ & NA & $23.26 \pm 3.35$ & $22.34 \pm 3.09$ \\
\hline
\end{tabular}

NA, not applicable.

\section{BASDAI disease activity index, BASFI functional index and BASMI measurement index of patients with AS}

We further analyzed the BASDAI disease activity index, the BASFI functional index and the BASMI measurement index of the patients before and after treatment. Results showed that the treatment significantly decreased the BASDAI disease activity index $(2.07 \pm 0.71)$, the BASFI functional index $(2.16 \pm 0.82)$ and the BASMI measurement index $(3.13 \pm 1.22)$ in the patients after treatment compared to indexes in patients in the active stage of AS (4.12 \pm 1.91 for BASDAI, $4.92 \pm 1.31$ for BASFI, and $5.81 \pm 2.92$ for BASMI $)(\mathrm{P}<0.05$, Table 2)

Table 2. BASDAI, BASFI, and BASMI scores before and after treatment of patients with AS.

\begin{tabular}{l|c|c|c}
\hline Index & Control & Before treatment & After treatment \\
\hline BASDAI & NA & $4.12 \pm 1.91$ & $2.07 \pm 0.71^{*}$ \\
\hline BASFI & NA & $4.92 \pm 1.31$ & $2.16 \pm 0.82^{*}$ \\
\hline BASMI & NA & $5.81 \pm 2.92$ & $3.13 \pm 1.22^{*}$ \\
\hline
\end{tabular}

NA, not applicable; $* \mathrm{P}<0.05$ on comparison with values before treatment.

\section{Levels of Th1 cytokines IFN- $\gamma$ and TNF- $\alpha$ in patients with AS before and after treatment}

ELISA was used to quantify the levels of the Th1 cytokines, IFN- $\gamma$ and TNF- $\alpha$, in serum from patients with AS. Results showed elevated serum level of IFN- $\gamma(128.6 \pm 19.9 \mathrm{pg} /$ $\mathrm{mL})$ and TNF- $\alpha(145.2 \pm 22.5 \mathrm{pg} / \mathrm{mL})$ in patients with active AS compared to the levels in the control individuals $(63.2 \pm 10.1 \mathrm{pg} / \mathrm{mL}$ for IFN- $\gamma, 87.5 \pm 13.2 \mathrm{pg} / \mathrm{mL}$ for TNF- $\alpha)(\mathrm{P}<0.05)$. After treatment, serum levels of IFN- $\gamma(95.8 \pm 15.6 \mathrm{pg} / \mathrm{mL})$ and TNF- $\alpha(123.1 \pm 20.1 \mathrm{pg} / \mathrm{mL})$ decreased $(\mathrm{P}<0.05)$ compared to those of patients with active AS before treatment (Figure 1).

\section{Levels of Th2 cytokines IL-4 and IL-10 in patients before and after treatment}

ELISA was used to quantify the levels of Th2 cytokines, IL-4 and IL-10. Results revealed significantly low levels of serum IL-4 $(65.1 \pm 12.5 \mathrm{pg} / \mathrm{mL})$ and IL-10 (72.3 $\pm 15.6 \mathrm{pg} /$

Genetics and Molecular Research 16 (1): gmr16019322 
$\mathrm{mL})$ in patients with active AS than in the control group $(129.6 \pm 29.8 \mathrm{pg} / \mathrm{mL}$ for IL-4, 158.6 $\pm 30.2 \mathrm{pg} / \mathrm{mL}$ for IL-10) $(\mathrm{P}<0.05)$. After treatment, serum levels of IL-4 $(92.3 \pm 13.9 \mathrm{pg} /$ $\mathrm{mL})$ and IL-10 $(100.6 \pm 16.3 \mathrm{pg} / \mathrm{mL})$ were enhanced in the patients compared to that before treatment $(\mathrm{P}<0.05)$ (Figure 2).

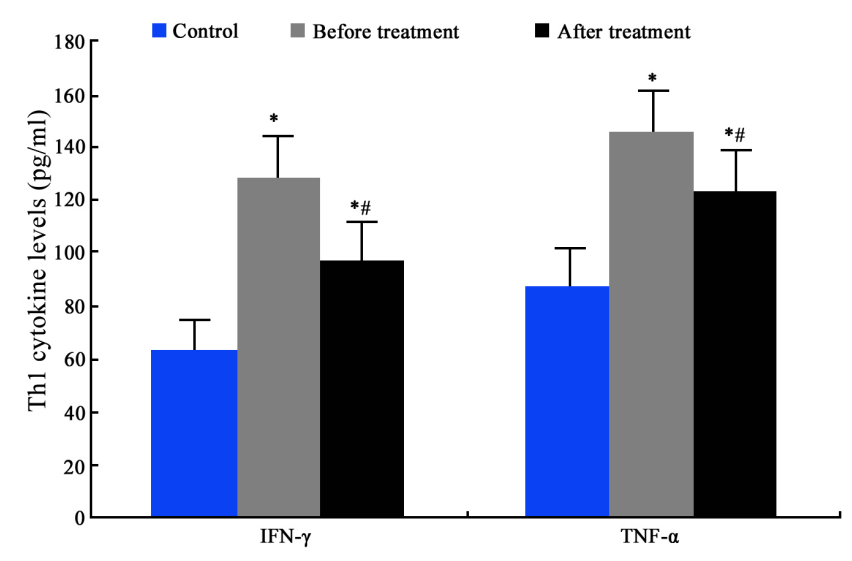

Figure 1. Serum levels of Th1 cytokines, IFN- $\gamma$ and TNF- $\alpha$. $* \mathrm{P}<0.05$ compared with levels in the control group; ${ }^{\#} \mathrm{P}<0.05$ compared with the levels before treatment. Data are presented as means \pm SD.

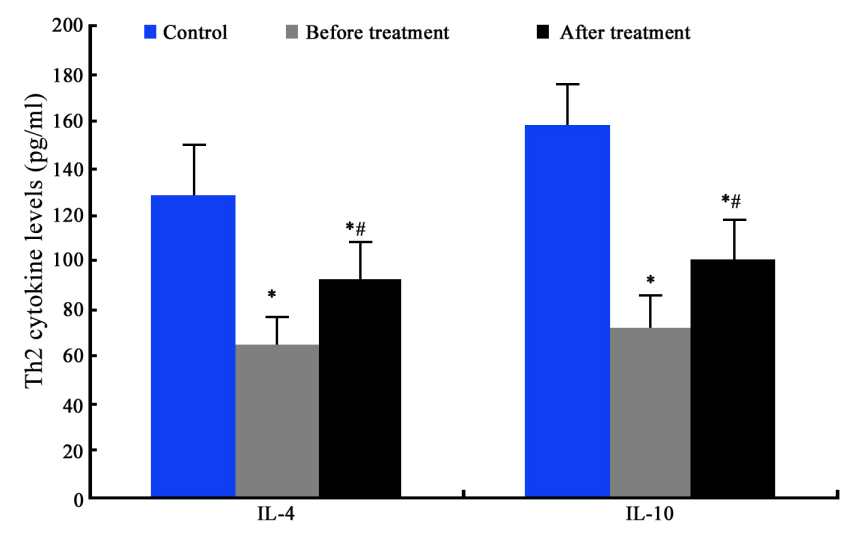

Figure 2. Serum levels of Th2 cytokines, IL-4 and Il-10 in patients with AS. $* \mathrm{P}<0.05$ compared with levels in the control group; ${ }^{\mathrm{P}}<0.05$ compared with the levels before treatment.

\section{Correlation between Th1 cytokine levels and clinical index of patients with AS}

Correlation analysis was used to study the relationship between levels of the Th1 cytokines IFN- $\gamma /$ TNF- $\alpha$ and the BASDAI disease activity index, the BASFI functional index, the BASMI measurement index, and the ESR and CRP levels in the patients before and after treatment. Results showed positive correlation between IFN- $\gamma /$ TNF- $\alpha$ levels and the BASDAI disease activity index, the BASFI functional index and the BASMI measurement index $(\mathrm{P}<$ 0.05 in all pairs) but not with CRP or ESR (P > 0.05) (Table 3). 
Table 3. Correlation analysis between Th1 cytokine levels and clinical indexes in patients with AS.

\begin{tabular}{l|c|c|c|c|c|c}
\hline & & BASDAI & BASFI & BASMI & ESR & CRP \\
\hline IFN- $\gamma$ & r value & 0.702 & 0.815 & 0.562 & 0.014 & 0.153 \\
\hline TNF- $\alpha$ & r value & 0.639 & 0.789 & 0.958 & 0.016 & 0.216 \\
\hline & $\mathrm{P}$ & $<0.05$ & $<0.05$ & $<0.05$ & $>0.05$ & $>0.05$ \\
\hline
\end{tabular}

\section{Correlation between Th2 cytokine levels and the clinical index of the patients}

Correlation analysis was used to study the correlation between levels of the Th2 cytokines IL-4 or IL-10 and the BASDAI disease activity index, the BASFI functional index, the BASMI measurement index, and levels of ESR and CRP in the patients before and after treatment for AS. Results showed negative correlation between IL-4 or IL-10 levels and the BASDAI disease activity index, the BASFI functional index, and the BASMI measurement index $(\mathrm{P}<0.05$ in all pairs) but not with CRP or ESR $(\mathrm{P}>0.05)$ (Table 4).

Table 4. Correlation analysis between Th2 cytokine levels and clinical indexes in patients with AS.

\begin{tabular}{l|l|c|c|c|c|c}
\hline & & BASDAI & BASFI & BASMI & ESR & CRP \\
\hline IL-4 & r value & -0.517 & -0.815 & -0.679 & 0.018 & 0.327 \\
\hline IL-10 & r value & -0.728 & -0.652 & -0.562 & 0.011 & 0.215 \\
\hline & P & $<0.05$ & $<0.05$ & $<0.05$ & $>0.05$ & $>0.05$ \\
\hline
\end{tabular}

\section{DISCUSSION}

As a common autoimmune disease, AS has a complicated pathogenesis mechanism, which is closely related to the body's immune functions (Gönül et al., 2016). Helper T cells (Th) can be sub-divided into different sub-populations, which play important roles in the pathogenesis and progression of several autoimmune diseases. Among these cells, Th1 and Th2 secrete cytokines, imbalance in the levels of which might be critical for the development of autoimmune diseases (Bautista-Caro et al., 2014). Normally, the body balances the numbers of Th1 and Th2 to maintain homeostasis. In various autoimmune diseases such as allergic enteritis and asthma-related rheumatoid arthritis, an imbalance of Th1/Th2 is observed. TNF- $\alpha$ is upregulated when the number of Th1 cells increase, thus hindering the normal growth of bone and cartilage and causing chronic inflammation. The elevation of TNF- $\alpha$ secretion is thus believed to be an important factor in the pathogenesis of autoimmune diseases, including rheumatoid arthritis and AS. Therefore, the biological therapy of AS frequently utilizes TNF- $\alpha$ antagonists for pain relief (Lee et al., 2016). Cytokines released from Th1 and Th2 cells can antagonize each other and inhibit the Th reactions and the release of related factors. The Th1 cytokine IFN- $\gamma$ can antagonize the secretion of Th2 cytokines IL-4 and IL-10, which further inhibits the Th2 reaction (van der Weijden et al., 2016). This study revealed significantly lower levels of serum IL-4 and IL-10 in patients with active AS, who also had higher levels of TNF- $\alpha$ and IFN- $\gamma$, thereby indicating that imbalance in Th1/Th2 cell numbers might be involved in the pathogenesis of AS.

Major therapies for AS include the use of non-steroid anti-inflammatory medications to manage symptoms and control inflammation. However, these therapies lack a systemic treatment plan. In addition, biological therapy is expensive and is an economic burden for the

Genetics and Molecular Research 16 (1): gmr16019322 
patients. The use of glucocorticoids has therefore become a feasible alternative for treatment of AS and it has been widely accepted regarding its efficacy (Kisacik et al., 2016b). However, effective evaluation criteria for the disease condition and prognosis of AS are still lacking. This study determined the levels of Th1 and Th2 cytokines in patients with AS before and after treatment and analyzed the correlation of the levels of the cytokines with clinical symptoms. Our results demonstrated a positive correlation between TNF- $\alpha /$ IFN- $\gamma$ levels and the disease activity indexes and a negatively correlation with IL-4/IL-10 levels, but not with CRP or ESR levels. These results showed that the dynamics of Th1/Th2 cytokines could be correlated with the progression of AS and be used for prognostic analysis. However, the exact mechanisms by which Th1/Th2 cytokines regulate the pathogenesis of AS was not addressed in our study, which forms the main limitation of this study as well as its future prospective.

In summary, increased expression of Th1 cytokines and decreased expression of Th2 cytokines in the peripheral blood of patients with AS can reflect the disease activity condition.

\section{Conflicts of interest}

The authors declare no conflict of interest.

\section{ACKNOWLEDGMENTS}

We thank the anonymous reviewers for reviewing this manuscript.

\section{REFERENCES}

Almasi S, Aslani S, Poormoghim H, Jamshidi AR, et al. (2016). Gene expression profiling of toll-like receptor 4 and 5 in peripheral blood mononuclear cells in rheumatic disorders: ankylosing spondylitis and rheumatoid arthritis. Iran. J. Allergy Asthma Immunol. 15: 87-92.

Arikan S, Gokmen F, Ersan I, Akbal A, et al. (2016). Effect of systemically used anti-tumor necrosis factor-alpha medication on the corneal epithelium and stroma of patients with ankylosing spondylitis. Ocul. Immunol. Inflamm. 5: 1-6. http://dx.doi.org/10.3109/09273948.2015.1107592

Bautista-Caro MB, Arroyo-Villa I, Castillo-Gallego C, de Miguel E, et al. (2014). Decreased frequencies of circulating follicular helper T cell counterparts and plasmablasts in ankylosing spondylitis patients Naïve for TNF blockers. PLoS One 9: e107086. http://dx.doi.org/10.1371/journal.pone.0107086

Colombo D, Chimenti S, Grossi PA, Marchesoni A, et al. (2015). Prevalence of acute and chronic viral seropositivity and characteristics of disease in patients with psoriatic arthritis treated with cyclosporine: a post hoc analysis from a sex point of view on the observational study of infectious events in psoriasis complicated by active psoriatic arthritis. Clin. Cosmet. Investig. Dermatol. 9: 1-7.

Fischer CP, Emary PC and Taylor JA (2015). Presumptive late-onset ankylosing spondylitis simulating osteoblastic skeletal metastasis in a patient with a history of prostate carcinoma: a diagnostic challenge. J. Chiropr. Med. 14: 259-264. http://dx.doi.org/10.1016/j.jcm.2015.06.005

Gönül Y, Genç A, Ahsen A, Bal A, et al. (2016). The effects of IL-18BP on mRNA expression of inflammatory cytokines and apoptotic genes in renal injury induced by infrarenal aortic occlusion. J. Surg. Res. 202: 33-42. http://dx.doi. org/10.1016/j.jss.2015.12.026

Gracey E, Yao Y, Green B, Qaiyum Z, et al. (2016). Sexual dimorphism in the Th17 signature of ankylosing spondylitis. Arthritis Rheumatol. 68: 679-689. http://dx.doi.org/10.1002/art.39464

Hoxha A, Calligaro A, Tonello M, Ramonda R, et al. (2016). The clinical relevance of early anti-adalimumab antibodies detection in rheumatoid arthritis, ankylosing spondylitis and psoriatic arthritis: A prospective multicentre study. Joint Bone Spine 83: 167-171. http://dx.doi.org/10.1016/j.jbspin.2015.04.020

Kisacik B, Pamuk ON, Onat AM, Erer SB, et al. (2016a). Characteristics predicting tuberculosis risk under tumor necrosis factor-alpha inhibitors: report from a large multicenter cohort with high background prevalence. J. Rheumatol. 43:

Genetics and Molecular Research 16 (1): gmr16019322 
524-529. http://dx.doi.org/10.3899/jrheum.150177

Kisacik P, Unal E, Akman U, Yapali G, et al. (2016b). Investigating the effects of a multidimensional exercise program on symptoms and antiinflammatory status in female patients with ankylosing spondylitis. Complement. Ther. Clin. Pract. 22: 38-43. http://dx.doi.org/10.1016/j.ctcp.2015.11.002

Lee YH, Kim EY, Jeong W, Kim YG, et al. (2016). Complete remission of nephrotic syndrome without resolution of amyloid deposit after anti-tumor necrosis factor alpha therapy in a patient with ankylosing spondylitis. J. Clin. Rheumatol. 22: 86-88. http://dx.doi.org/10.1097/RHU.0000000000000356

Liang H, Li WR, Zhang H, Tian X, et al. (2015). Concurrent intervention with exercises and stabilized tumor necrosis factor inhibitor therapy reduced the disease activity in patients with ankylosing spondylitis: a meta-analysis. Medicine (Baltimore) 94: e2254. http://dx.doi.org/10.1097/MD.0000000000002254

Lindström U, Bremander A, Haglund E, Bergman S, et al. (2016a). Back pain and health status in patients with clinically diagnosed ankylosing spondylitis, psoriatic arthritis and other spondyloarthritis: a cross-sectional population-based study. BMC Musculoskelet. Disord. 17: 106. http://dx.doi.org/10.1186/s12891-016-0960-8

Lindström U, Forsblad-d'Elia H, Askling J, Kristensen LE, et al. (2016b). Perinatal characteristics, older siblings, and risk of ankylosing spondylitis: a case-control study based on national registers. Arthritis Res. Ther. 18: 16. http://dx.doi. org/10.1186/s13075-016-0917-1

Qian SJ, Ye XS, Chen WS and Li WL (2016). Missed diagnosis of oesophageal perforation in ankylosing spondylitis cervical fracture: Two case reports and literature review. J. Int. Med. Res. 44: 170-175. http://dx.doi. org/10.1177/0300060515614230

Roberts AR, Vecellio M, Chen L, Ridley A, et al. (2016). An ankylosing spondylitis-associated genetic variant in the IL23RIL12RB2 intergenic region modulates enhancer activity and is associated with increased Th1-cell differentiation. Ann. Rheum. Dis. 75: 2150-2156. http://dx.doi.org/10.1136/annrheumdis-2015-208640

van der Weijden MA, van Denderen JC, Lems WF, Nurmohamed MT, et al. (2016). Etanercept increases bone mineral density in ankylosing spondylitis, but does not prevent vertebral fractures: results of a prospective observational cohort study. J. Rheumatol. 43: 758-764. http://dx.doi.org/10.3899/jrheum.150857

Wang C, Liao Q, Hu Y and Zhong D (2015). T lymphocyte subset imbalances in patients contribute to ankylosing spondylitis. Exp. Ther. Med. 9: 250-256.

Wang J, Zhao Q, Wang G, Yang C, et al. (2016). Circulating levels of Th1 and Th2 chemokines in patients with ankylosing spondylitis. Cytokine 81: 10-14. http://dx.doi.org/10.1016/j.cyto.2016.01.012

Yu HC, Lu MC, Huang KY, Huang HL, et al. (2015). Sulfasalazine treatment suppresses the formation of HLA-B27 heavy chain homodimer in patients with ankylosing spondylitis. Int. J. Mol. Sci. 17: 17. http://dx.doi.org/10.3390/ ijms17010046

Zou YC, Yang XW, Yuan SG, Zhang P, et al. (2016). Celastrol inhibits prostaglandin E2-induced proliferation and osteogenic differentiation of fibroblasts isolated from ankylosing spondylitis hip tissues in vitro. Drug Des. Devel. Ther. 10: 933-948.

Genetics and Molecular Research 16 (1): gmr16019322 